\title{
The steady-state development of splenic dendritic cells
}

\author{
P Sathe $^{1}$ and K Shortman ${ }^{1}$
}

Dendritic cells (DCs) are a heterogenous population of cells that can be grouped into the conventional DCs (cDCs) and plasmacytoid DCs (pDCs), or interferon-producing cells. pDCs are thought to develop in the bone marrow and migrate to the periphery as mature cells. In contrast, CDC precursors are thought to migrate to the periphery, where they further differentiate into $\mathrm{CDCs}$. In the case of migratory $\mathrm{CDCs}$, these precursors are thought to be monocytes, whereas resident cDCs derive from a different precursor. Recent activity on this subject has shed some light on the precursors that differentiate into resident $\mathrm{CDCs}$ and $\mathrm{pDCs}$, but often with conflicting findings. Here, we review some of these findings and discuss some of the outstanding issues in the field.

In 1973, Steinman and Cohn ${ }^{1}$ described "novel cells (which) can assume a variety of branching forms, and constantly extend and retract many fine cell processes." They named these cells "dendritic cells (DCs)," after their branches or dendrites. These DCs have been shown to be the most potent of the group of cells known as antigen-presenting cells. DCs alone are able to stimulate naive T cells, both $\mathrm{CD} 4^{+}$and $\mathrm{CD}^{+} \mathrm{T}$ cells.

It was initially assumed that DCs were essentially a single cell type that was distributed through various locations in the body. However, it has become clear over the years that DCs are a diverse population and can be separated into distinct subsets, ${ }^{2}$ the common denominator being their ability to efficiently internalize, process, and present antigen to T cells. Further, these DC subsets have different functional roles within the immune system. The immune system has evolved a range of responses, tailored not only to the pathogen in question, but also the site of infection. The immune system responds differently to viruses than it does to bacteria. ${ }^{3}$ DCs from the gut induce the production of IgA that protects the host against commensal bacteria, ${ }^{4}$ yet if these DCs reacted to bacteria in the same way as splenic DCs, the result would be an immunopathology. When and how in our evolutionary history the different DC subsets arose is not known, but it seems likely that the evolution of different DC subsets has allowed the immune system to recognize a range of pathogens in different areas of the body and to respond appropriately.

\section{DENDRITIC CELL SUBTYPES}

Although we focus here on those DC subtypes that can be found in steady-state adult mice, it is important to note the existence of another type of DCs that develops under conditions of infection or inflammation. ${ }^{6}$ These "inflammatory DCs" are derived from monocytes, and their development can be induced by "danger signals" that mimic infection, such as Toll-like receptor ligands. ${ }^{7}$

Those DC subtypes that develop under steady-state conditions can be described in a hierarchical fashion, by classification and subclassification into different groups. The initial division we will make here is between conventional DCs (cDCs) or plasmacytoid pre-DCs (pDCs).

Plasmacytoid pre-DCs circulate through the blood and are found in both the lymphoid organs and in the peripheral tissues. They exist in the steady state as rounded cells, and it is only on activation that they develop dendritic processes. When activated, pDCs secrete large amounts of type I interferon and are hence also known as interferon-producing cells. ${ }^{8}$ pDCs are thought to be important in antiviral immune responses.

Two $\mathrm{CDC}$ groups can be described based on their regular place of residence. One-the "migratory DCs" - are stationed in the peripheral tissues of the body-for example, the skin or lungs. ${ }^{9}$ Migratory cDCs constantly sample these tissues, which are most directly exposed to the external environment, for any sign of invading pathogens. On activation, they migrate with their antigen through lymph to the draining lymph node, where they encounter naive $\mathrm{T}$ cells, and activate immune responses. ${ }^{10-12}$

1The Walter and Eliza Hall Institute of Medical Research, Melbourne, Victoria, Australia. Correspondence: P Sathe (sathe@wehi.edu.au)

Received 28 June 2008; accepted 5 August 2008; published online 10 September 2008. doi:10.1038/mi.2008.56 
There is a constitutive low incidence of migration to the draining lymph nodes even in the steady state. ${ }^{13-15}$ The DCs that migrate under these steady-state conditions may be involved in the maintenance of tolerance when they encounter $\mathrm{T}$ cells; for example, oral tolerance is dependent on constitutive migration by mucosal DCs to the mesenteric lymph node. ${ }^{16}$ Migratory DCs, regardless of whether they migrate in the steady state or on inflammation, acquire a relatively mature phenotype, expressing higher levels of major histocompatibility complex (MHC) class II and costimulatory molecules by the time they arrive in the lymph nodes. They can therefore be distinguished from lymphoid tissue-resident DCs, discussed below, which exist in the peripheral lymphoid organs in an immature state. ${ }^{17}$ The subsets, function, and the development of these migratory DCs are discussed elsewhere in this issue.

The second group of cDCs are the lymphoid tissue-resident DCs, which are nonmigratory and execute their antigen collection, processing, and presentation functions within the lymphoid organ. These $\mathrm{cDCs}$ can be found in all lymphoid organs in the steady state ${ }^{17}$ and, in the absence of migratory DCs, are the major DC population in the spleen. In addition to their likely role in mediating tolerance, these $\mathrm{cDCs}$ constantly sample the blood and lymph for pathogens. Found in the steady state as immature DCs, these resident $\mathrm{CDCs}$ upregulate the expression of MHC and costimulatory molecules on inflammation or pathogen encounter and become efficient activators of T-cells. ${ }^{17}$

The final division we will make is of the resident cDCs, based-in the mouse-on their expression of the CD8 $\alpha$ homodimer and $\mathrm{CD} 4$ molecules, into $\mathrm{CD} 8^{+} \mathrm{CD} 4^{-}, \mathrm{CD} 8^{-} \mathrm{CD} 4^{+}$, and $\mathrm{CD} 8{ }^{-} \mathrm{CD} 4^{-}$cDCs. ${ }^{18,19}$ The relevance of $\mathrm{CD} 8$ and $\mathrm{CD} 4$ expression on these DCs is unclear, and other markers, such as $\mathrm{CD} 11 \mathrm{~b}$, Sirp $\alpha$, and CD24, can provide a related division of subsets. ${ }^{18,20,21}$

$\mathrm{CD}^{-}{ }^{-} \mathrm{CD} 4^{+}$and $\mathrm{CD} 88^{-} \mathrm{CD} 4^{-}$cDCs differ somewhat in their profile of chemokine production, but otherwise seem to be functionally similar. ${ }^{22}$ We shall here refer to both subsets as $\mathrm{CD} 8^{-}$ cDCs. However, $\mathrm{CD} 8^{-}$and $\mathrm{CD} 8^{+}$cDCs differ markedly in cytokine production, ${ }^{23}$ antigen processing, and Toll-like receptor expression. When activated, $\mathrm{CD} 8^{+} \mathrm{cDCs}$ are the most efficient producers of interleukin-12, thus initiating inflammatory Th1 responses. ${ }^{24,25}$ They are also the most efficient DCs at a process known as cross-presentation, ${ }^{26,27}$ by which exogenously derived antigen is diverted to the MHC class I presentation pathway. These characteristics make $\mathrm{CD} 8{ }^{+} \mathrm{cDCs}$ the primary inducers of cytotoxic T-cell responses to viral infections. ${ }^{28}$

Given their efficiency at cross-presentation, $\mathrm{CD} 8{ }^{+} \mathrm{cDCs}$ can present tissue-derived self-antigen to naive $\mathrm{CD} 8^{+} \mathrm{T}$ cells. Further, even in the steady state, $\mathrm{CD} 8^{+} \mathrm{cDCs}$ can be found in the T-cell areas of the lymphoid tissue. ${ }^{29}$ In contrast, $\mathrm{CD} 8^{-} \mathrm{cDCs}$ are normally found within the marginal zones of lymphoid tissue and migrate to the $\mathrm{T}$-cell areas only on activation. ${ }^{25,30-32}$ Hence, $\mathrm{CD} 8^{+} \mathrm{cDCs}$ are in contact with $\mathrm{T}$ cells in the steady state, making them both ideally situated and functionally equipped to mediate tolerance to self-antigen. ${ }^{33}$

We will here focus on the ontogeny of those DCs found in the steady-state spleen; that is, lymphoid tissue-resident cDCs and pDCs. Detailed information on the development of these particular DC subtypes may serve as a background and a point of comparison for the development of DCs of the mucosal tissues. For convenience, and as the migratory $\mathrm{CDC}$ subsets are discussed elsewhere, we shall here refer to resident cDCs as simply "cDCs," and use the spleen as a model of their development in other lymphoid organs.

\section{IN VITRO MODELS OF DC DEVELOPMENT}

DCs are rare cells, and many studies are reliant on the generation of DCs in culture. This has largely been performed by culturing monocytes with granulocyte macrophage colony-stimulating factor (GM-CSF) and interleukin-4. The DCs derived from these cultures lack the heterogeneity of the DCs found in lymphoid tissue and are thought to be the in vitro equivalent of inflammatory, rather than steady-state, DCs. ${ }^{34}$ In fact, mice lacking GM-CSF or GM-CSF receptor (GM-CSFR) show no defects in the development of steady-state splenic DCs. ${ }^{35,36}$ Further, mice lacking functional macrophage-colony stimulation factor (M-CSF) or MCSFR, which have defects in monocytes, show no specific defect in steady-state DC development, ${ }^{37}$ suggesting monocytes are not the true in vivo precursors of resident DCs.

In contrast, mice lacking fms-like tyrosine kinase-3 (flt3) ligand (FL) show a severe reduction in their DC levels. ${ }^{38} \mathrm{~A}$ similar defect is seen in mice in which the hematopoietic compartment lacks expression of STAT3, ${ }^{39}$ a transcription factor involved in flt3 signaling. Given that flt 3 expression on precursors is an indicator of DC precursor activity, it seems reasonable to conclude that FL, rather than GM-CSF or interleukin-4, is the limiting cytokine involved in steady-state DC development.

Indeed, bone marrow cultured with FL gives rise to DCs with all the heterogeneity of those found in the spleen. ${ }^{40}$ Although the cDCs in FL-stimulated cultures lack surface expression of the CD8 molecule on which the subsets are commonly segregated, the heterogenous expression of other molecules differentially expressed on the cDC subsets is maintained, and these DCs have been demonstrated to be functionally equivalent to their splenic counterparts. ${ }^{41}$ Two precursors have been isolated from the FL culture system, discussed below, which are equivalent to bone marrow-derived precursors in their differentiation potential. ${ }^{42}$ The pathway of DC development in these cultures is therefore likely to model an in vivo process, making the FL culture system an appropriate in vitro system by which to study steady-state DC development.

It should be noted that the FL culture system is not without its limitations. First, in these cultures, the DC developmental process-which in vivo has stages in both the bone marrow and spleen - takes place within the one culture vessel. Second, the levels of FL used in these cultures are well above those found in the steady state. Hence, although the DC developmental pathway in these cultures may model an in vivo process, the importance of certain pathways may be exaggerated.

\section{DEVELOPMENT INTHE SPLEEN: THE LATTER STAGES}

Although DCs differentiate from bone marrow-derived cells, few cDCs are thought to develop completely within the bone marrow itself. cDCs are not found in significant numbers in either the 
bone marrow or blood, suggesting that $\mathrm{cDC}$ precursors, rather than cDCs, migrate to the spleen and other peripheral lymphoid tissues, where they undergo the final stages of differentiation. This situation is therefore similar to that of migratory DCs in the skin, where monocytes migrate to the epidermis after DC depletion before differentiating into Langerhans cells. ${ }^{43,44}$

pDCs, on the other hand, can be found in significant numbers in the bone marrow and blood, ${ }^{45}$ suggesting that like $\mathrm{B}$ cells, they differentiate within the bone marrow before migration to the spleen. Although there are changes in pDC surface markers, indicating further maturation in the spleen, pDCs are already recognizable and functional within the bone marrow and blood.

The frequency with which cDCs require replenishment from bone marrow precursors varies with different DC subtypes. For example, on bone marrow transplant with a congeneic donor, Langerhans cells are replaced slowly, indicating that they may have long half-lives and are replenished by a skin-resident precursor. ${ }^{44}$ In contrast, as determined by continuous 5-bromo-2deoxyuridine labeling in vivo, cDCs appeared to have a short half-life of 1.5-3 days in the spleen. ${ }^{46}$ It should be noted that in these experiments, this half-life was determined by measuring the loss of unlabeled DCs. This was based on the assumption that DCs were end-stage nondividing cells, so that any 5-bromo2-deoxyuridine-labeled DCs should have been the product of dividing precursors. However, it has since been shown that splenic cDCs are capable of division - at any given point, up to $5 \%$ are in cell cycle. ${ }^{47} \mathrm{CD} 8{ }^{-} \mathrm{cDCs}$, for example, proliferate in response to lymphotoxin $\beta,{ }^{47}$ and FL has recently been shown to promote cDC expansion in the spleen. ${ }^{48}$ Hence, the half-life indicated refers to the average time to death or division, rather than life span.

Experiments with parabiotic mice indicated that after 6 weeks of shared circulation, the splenic $\mathrm{CD} 8^{-} \mathrm{cDCs}$ failed to equilibrate, the majority being of host origin. ${ }^{47}$ In light of this data and the observation that DCs were capable of division, it was proposed that the spleen was capable of maintaining DC homeostasis without further precursor input from the bone marrow. However, a subsequent study using parabiotic mice demonstrated that this failure of splenic cDCs to equilibrate was due to the fact that $\mathrm{CDC}$ precursors were rapidly cleared from circulation, and thus could not equilibrate in parabiotic mice. ${ }^{49}$ This study also demonstrated that, whereas the half-life of the CDC population and its progeny in spleen was actually $5-7$ dayslonger than that estimated by 5-bromo-2-deoxyuridine-labeling experiments $-\mathrm{cDCs}$ did require constant replenishment from bone marrow-derived precursors.

So what might be the immediate precursors of splenic cDCs? It was suggested that a monocyte-like cell might fulfill this role. ${ }^{50}$ However, a cDC-restricted precursor, the pre-cDC, has been isolated from the mouse spleen. ${ }^{51}$ These pre-cDCs were clearly distinct from monocytes, which show little splenic cDC precursor activity in the steady state. The pre-cDCs appeared to be restricted to the formation of $\mathrm{cDCs}$, giving rise to both $\mathrm{CD}^{+}$ and $\mathrm{CD} 8^{-} \mathrm{cDCs}$ on in vivo transfer, but no other lineages, not even pDCs. Pre-cDCs, which were already CD11c ${ }^{+}$, but did not yet express surface MHC class II, were found to be heterogenous in their expression of $\mathrm{CD} 24$. The $\mathrm{CD} 24^{\text {hi }}$ pre-cDCs were restricted to the formation of $\mathrm{CD} 8{ }^{+} \mathrm{cDCs}$, whereas the $\mathrm{CD} 24^{\text {low }}$ fraction produced only $\mathrm{CD} 8^{-} \mathrm{CDCs}$, indicating that much subset commitment had already occurred by this late precursor stage. Pre-cDCs divided only one to three times before differentiating into $\mathrm{CDCs}$, indicating that these precursors are the last stage in the $\mathrm{CDC}$ differentiation pathway, and are likely to be the type of precursor that migrates from the bone marrow to spleen.

\section{STAGES OF DC DEVELOPMENT INTHE BONE MARROW: THE EARLY PRECURSORS}

During hematopoiesis, multipotent cells undergo a process of increasing commitment, differentiating into precursors increasingly biased toward a particular type of blood cell, until they are committed to the formation of that lineage..$^{52}$ An initial step in this process was shown to be the differentiation of multipotent progenitors into common myeloid progenitors $(\mathrm{CMPs})^{53}$ and common lymphoid progenitors (CLPs). ${ }^{54}$

It was originally considered self-evident that DCs were of myeloid origin ${ }^{55}$-after all, they express a variety of myeloid markers, such as M-CSFR. ${ }^{56}$ Further, the predominant model for the generation of DCs thus far has involved the culture of monocytes - a population of myeloid origin - with GM-CSF and interleukin $-4,{ }^{57}$ the two cytokines traditionally involved in the differentiation of myeloid cells. This concept was shown to be limited after a thymic precursor, restricted to lymphoid development, was shown to have DC potential. ${ }^{58}$ DC development was found to be more flexible than suggested by the lymphoid vs. myeloid model of hematopoiesis, when potential for each of the splenic DC subtypes was demonstrated in both CLPs and CMPs. ${ }^{59-61}$ Thus far, the only known defining characteristic of an early precursor with DC potential is its expression of flt3. ${ }^{62,63}$

\section{INTERMEDIATE STAGES OF CDC DEVELOPMENT IN BONE MARROW}

As little commitment to the DC lineage was evident at the early precursor stages, downstream precursors were examined for their DC precursor potential. A number of $\mathrm{CDC}$ precursors have been isolated till date (Figure 1). However, their relationship to one another and their relative contributions to steady-state cDCs remain unclear.

Two downstream precursors have been isolated from bone marrow that are capable of differentiating into cDCs. One, termed a common DC precursor (CDP), or pro-DC, was isolated as the inn $^{-} \mathrm{c}-\mathrm{kit}^{\mathrm{int}} \mathrm{flt}{ }^{+} \mathrm{M}-\mathrm{CSFR}^{+}$population of the bone marrow. ${ }^{42,64} \mathrm{CDPs}$ are largely restricted to the $\mathrm{DC}$ lineage and are able to give rise to pDCs and both $\mathrm{cDC}$ subsets both in vitro and in vivo, but no other lineages, beyond a low capacity to form macrophages. Clonal analysis in vitro found single CDPs that were able to form both pDCs and cDCs. Hence, the population was not entirely a heterogenous group composed of separate $\mathrm{CDC}$ and $\mathrm{pDC}$ precursors, but rather contained many single cells capable of forming both the steady-state DC splenic subsets. A similar precursor population was described in the FL bone marrow culture system named pro-DC, this popula- 


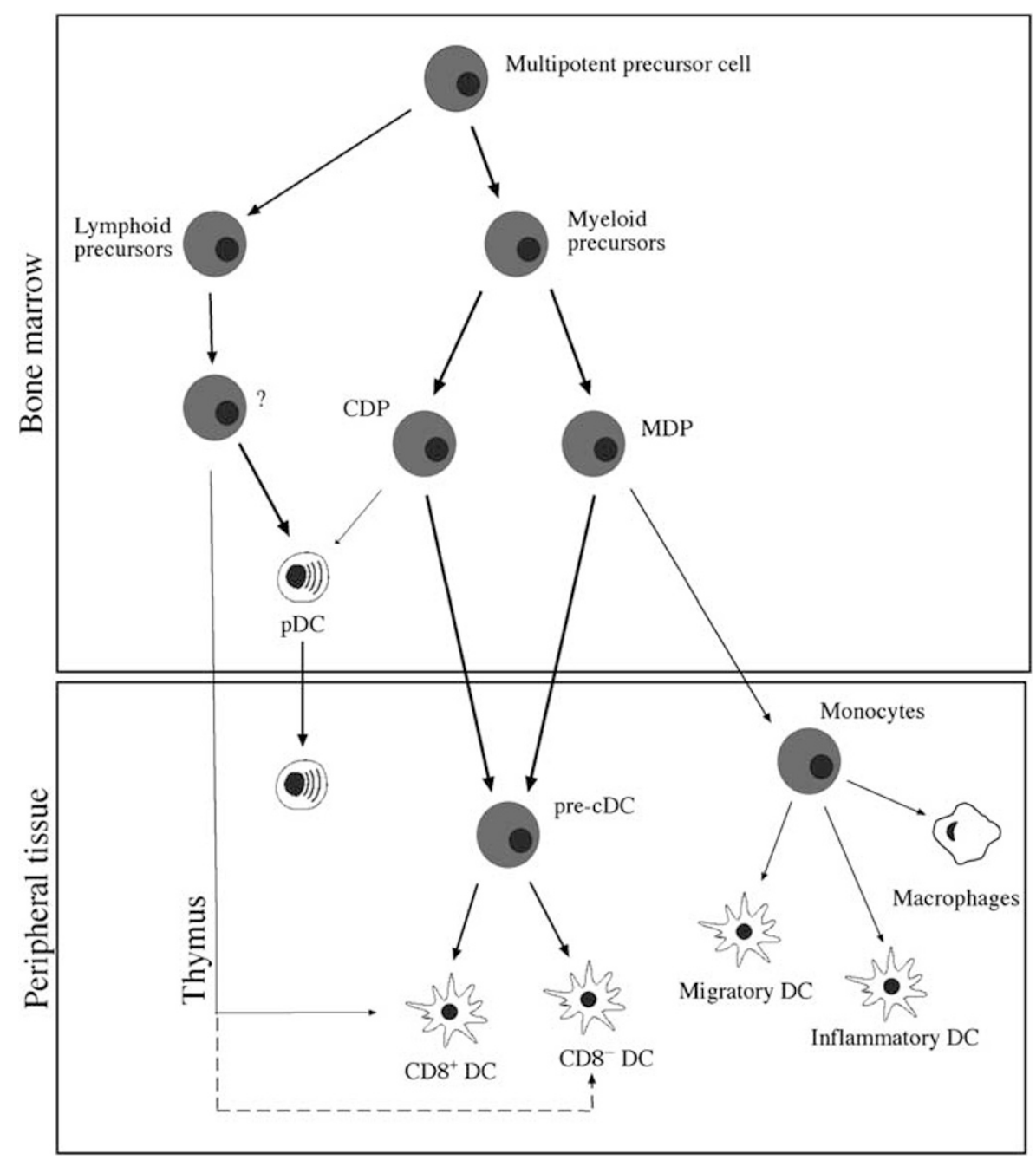

Figure 1 Dendritic cell (DC) precursors in the bone marrow and peripheral tissues, and their possible relationships with each other. It should be noted that although common lymphoid progenitors (CLPs) have been shown to have potential for differentiation to conventional dendritic cells (cDCs), this has only been demonstrated in the thymus thus far.

tion being isolated as $\operatorname{lin}^{-} \mathrm{c}-\mathrm{kit}^{\mathrm{int}} \mathrm{flt}{ }^{+} \mathrm{M}-\mathrm{CSFR}^{+} \mathrm{CD} 11 \mathrm{c}^{-} \mathrm{MHC}$ class $\mathrm{II}^{-}$. Like CDPs, these in vitro derived pro-DCs were largely DC restricted in both in vitro and in vivo assays, showing only a low macrophage potential. On a clonal level in vitro, single proDCs were found with potential for $\mathrm{pDCs}$ and both $\mathrm{cDC}$ subsets, thereby resembling the CDP population. Hence, at least in the FL culture system, the dominant source of the DC products is a CDP-like precursor. Whether this reflects the situation in vivo remains to be determined.

The other bone marrow $\mathrm{cDC}$ precursor, the macrophage DC precursor (MDP), was initially isolated as the $\operatorname{lin}^{-} \mathrm{c}^{-}$ $\mathrm{kit}^{+} \mathrm{CX}_{3} \mathrm{CR} 1^{+}$fraction of bone marrow. ${ }^{65}$ Unlike CDPs, MDPs could-in addition to their precursor potential for $\mathrm{CDC}$ - give rise to macrophages, but were devoid of potential for other lineages on in vivo transfer. MDPs were originally reported to have no $\mathrm{pDC}$ potential, but recent reports suggest that some pDCs may be produced. ${ }^{48}$ In clonal assays, single MDPs gave rise to both $\mathrm{cDCs}$ and macrophages, indicating that individual precursors existed with both macrophage and DC potential. ${ }^{65}$ However, these assays were performed with either GM-CSF or GM-CSF and FL, conditions which are likely to induce DC generation from monocytes. It thus remains to be determined whether the $\mathrm{CDC}$ s seen in these clonal assays here are the result of MDP-derived monocytes, ${ }^{66}$ responding to GM-CSF to form DCs or true steady-state DCs. On in vivo transfer, MDP could give rise to the steady-state splenic $\mathrm{CD} 8^{+}$and $\mathrm{CD} 8^{-} \mathrm{cDCs}$, but as this was performed with a bulk population, this may have been a result of heterogeneity with the MDP.

A recent study reported identical differentiation potential to MDP in the lin $^{-} \mathrm{M}-\mathrm{CSFR}^{+}$fraction of bone marrow. ${ }^{48} \mathrm{On}$ adoptive transfer, this population was found to have potential for both steady-state splenic CDC subsets. CDP_-defined as $\operatorname{lin}^{-}{ }^{-}-\mathrm{kit}^{\text {int }} \mathrm{flt}^{+}{ }^{+} \mathrm{M}-\mathrm{CSFR}^{+}-$must be contained within this $\mathrm{lin}^{-} \mathrm{M}-\mathrm{CSFR}^{+}$definition of MDP. Indeed, when MDPs were fractionated by expression of flt $3, \mathrm{CDC}$ potential was found to be concentrated within the flt $3^{+}$population, though to what extent is not specified. As these in vivo assays were performed with a bulk population of MDP, it has yet to be shown whether single MDPs are capable of differentiating into both macrophages and steady-state splenic DCs. Given that previous data 
have demonstrated that DC precursor potential is restricted to flt $3^{+}$cells, ${ }^{62}$ the possibility thus remains that MDPs are in fact a mixed population of macrophage precursors and CDP-like precursors-which fall within the flt $3^{+}$fraction-which gave rise to the steady-state splenic cDCs on adoptive transfer. Alternatively, if single MDPs do have both DC and macrophage potential, the population defined as flt $3^{-}$may in fact have expressed low levels of flt3, allowing them to respond to FL to form cDCs. Further studies are required to clarify the $\mathrm{CDC}$ and macrophage potential of individual MDP.

Although pro-DCs, the culture-derived precursors, seemed largely DC restricted, they retained some macrophage precursor activity by in vitro colony assays. Whether this is the result of a less differentiated precursor that could give rise to macrophages, $\mathrm{cDCs}$, and $\mathrm{pDCs}$, or whether the macrophages seen were a product of MDP-like precursors in the FL cultures is unclear. In the latter case, the pro-DCs would be a mixture of CDPs and those MDPs without $\mathrm{pDC}$ potential, perhaps reflecting the in vivo situation.

\section{PDC DEVELOPMENT}

As discussed above, pDCs develop within the bone marrow and migrate to peripheral tissues as differentiated cells. We have seen that the bone marrow contains DC precursors with potential for both pDCs and cDCs. pDCs may develop from these precursors, which give rise to both the $\mathrm{pDCs}$ and the $\mathrm{cDC}$ precursors that migrate to the spleen. Alternatively, pDCs may develop from an entirely separate precursor altogether.

Several lines of evidence point to more than one developmental pathway for pDCs. What these pathways might be has yet to be determined, although evidence suggests distinct routes through lymphoid and myeloid precursors. Some, but not all, pDCs from both the thymus and spleen were found to have D-J rearrangements in their immunoglobulin heavy genes, usually an indicator of derivation from a lymphoid precursor. ${ }^{67}$ However, it has been demonstrated that a proportion of pDCs derived from both common lymphoid and myeloid precursors had such rearrangements, ${ }^{68}$ indicating perhaps that this "lymphoid" molecular program could be activated even in pDCs of myeloid origin. As CMPs have some residual B-cell potential on in vivo transfer, ${ }^{69}$ it is possible that the definition of CMP does not exclude all lymphoid potential.

Another indicator of a lymphoid history is the expression of RAG1. In reporter mice that express green fluorescent protein (GFP) under the RAG1 promoter, two distinct $\mathrm{GFP}^{+}$and $\mathrm{GFP}^{-} \mathrm{pDC}$ populations were identified in the bone marrow and spleen, ${ }^{45}$ suggesting that there were two $\mathrm{pDC}$ populations with distinct developmental origins. Despite their apparent similarity, these populations were found to vary in their cytokine production, ability to stimulate T cells, and the nature of the T-cell responses they induced, suggesting that they were functionally distinct subsets.

If there are in fact two distinct lymphoid- and myeloid-orientated pathways to $\mathrm{pDC}$ development, it seems likely that the CDP-derived pDCs are the products of the myeloid pathway. This seems especially likely given that, on injection into the bone marrow, flt $3^{+}$myeloid, but not lymphoid precursors, could give rise to CDP.

Also, suggesting there are multiple pathways to $\mathrm{pDC}$ development, a recent study demonstrated that M-CSF, as well as FL, could drive pDC differentiation. ${ }^{70}$ Both CMPs and CLPs could respond to M-CSF to form pDCs, without any involvement of FL. However, whether this pathway is active in the steady state, or whether it reflects an inflammatory condition, is unclear. Further, whether both these pathways contribute to the pDC pool independently of one another, or whether they compete for the same precursors, has not yet been determined.

\section{FL INTHE PATHWAYTO DC DEVELOPMENT}

Mice lacking FL have decreased numbers of splenic DCs. ${ }^{38}$ Conversely, mice treated with FL have increased numbers of steady-state DCs. ${ }^{71,72}$ Flt3 expression defines DC potential in a precursor, ${ }^{62,63}$ and FL-stimulated bone marrow gives rise to DCs, functionally equivalent to those in the steady-state spleen. ${ }^{40,41,73}$ However, the question still remains as to whether FL is required for the direction of multipotent cells to the DC lineage or whether it acts as a proliferative and survival stimulus for DC precursors and immature DCs.

Enforced expression of flt3 in megakaryocyte-erythroid precursors, which ordinarily have no DC potential, restores some DC precursor activity, ${ }^{74}$ suggesting that flt 3 in this case is actually directing these cells into the DC lineage. If it were merely expanding responsive cells, we would expect the flt3-transfected megakaryocyte-erythroid precursors to expand, but continue along the erythroid lineage.

In contrast, parabiotic and chimera experiments with flt $3^{-1-}$ and $\mathrm{FL}^{-/-}$mice have demonstrated the need for flt3 at a late stage in differentiation, that is, after the bone marrow intermediate precursor stage. ${ }^{48}$ In this study, MDP did expand in response to FL administration, and flt $3^{-/-}$MDP showed impaired proliferation in wild-type spleens. However, flt $3^{-/-}$had normal numbers of MDP in the bone marrow. Further, when transferred into $\mathrm{FL}^{-/-}$recipients, MDP derived from wild-type mice showed no advantage in DC generation over Flt3-deficient MDP. Hence, the absence of flt3 had no effect on the generation of MDP or on their DC potential. The authors concluded that the effects of flt 3 on DC generation were restricted to regulating division in the periphery, rather than driving the generation of DC-restricted precursors. However, it is not yet clear that MDPs are the major contributors to splenic DCs, and it would be interesting to determine whether a defect is seen in the CDP compartment in the absence of FL-mediated effects. If commitment to the DC lineage is in fact FL independent, it would be interesting to determine which factors-either cell intrinsic or external-may be mediating this process.

\section{CONCLUSIONS}

Recent years have seen significant advancements in our understanding of splenic DC development, but many questions remain unanswered. Although we have some understanding of the precursors that can give rise to DCs, the exact nature of these precursors and their contribution to the DC pool have yet 
to be elucidated. Although it is evident that monocytes are not the steady-state precursors of splenic $\mathrm{cDCs}$, there appears to be a close relationship between cDCs and macrophages, and we do not know where these lineages diverge. Likewise, given the number of early precursors with DC potential, we do not know whether other pathways and other precursors also contribute to splenic DCs. Although it has been established that FL is the limiting cytokine in DC development, its exact role and the other factors involved in this process are unknown. The development of an in vitro system that models, albeit in an exaggerated fashion, the development of steady-state DCs will allow us to address some of these questions. However, to truly understand DC differentiation, we need to be able to track these precursors and their development in vivo. New techniques, for example, the introduction of unique genetic markers into individual cells"genetic barcoding," so to speak-may allow such analysis ( $\mathrm{T}$ Schumacher and S Naik, personal communication).

With a clearer understanding of splenic DC development in the steady state, we can better appreciate perturbations in this system, whether caused by deficiency in development or as a normal response to infections or inflammation. ${ }^{7}$ Of course, disruptions from the splenic steady state may be the norm for the mucosal system, with its constant contact with the external environment.

\section{DISCLOSURE}

The authors declared no conflict of interest.

() 2008 Society for Mucosal Immunology

\section{REFERENCES}

1. Steinman, R.M. \& Cohn, Z.A. Identification of a novel cell type in peripheral lymphoid organs of mice: morpholohy, quantitation, tissue distribution. J. Exp. Med. 137, 1142-1162 (1973).

2. Shortman, K. \& Liu, Y.J. Mouse and human dendritic cell subtypes. Nat. Rev. Immunol. 2, 151-161 (2002).

3. Netea, M.G., Van der Meer, J.W., Sutmuller, R.P., Adema, G.J. \& Kullberg, B.J. From the Th1/Th2 paradigm towards a Toll-like receptor/T-helper bias. Antimicrob. Agents Chemother. 49, 3991-3996 (2005).

4. Macpherson, A.J. \& Uhr, T. Induction of protective IgA by intestinal dendritic cells carrying commensal bacteria. Science 303, 1662-1665 (2004).

5. Duchmann, R., Schmitt, E., Knolle, P., Meyer zum Buschenfelde, K.H. \& Neurath, M. Tolerance towards resident intestinal flora in mice is abrogated in experimental colitis and restored by treatment with interleukin-10 or antibodies to interleukin-12. Eur. J. Immunol. 26, 934-938 (1996).

6. Serbina, N.V., Salazar-Mather, T.P., Biron, C.A., Kuziel, W.A. \& Pamer, E.G. TNF/iNOS-producing dendritic cells mediate innate immune defense against bacterial infection. Immunity 19, 59-70 (2003).

7. Nagai, Y. et al. Toll-like receptors on hematopoietic progenitor cells stimulate innate immune system replenishment. Immunity 24, 801-812 (2006).

8. Liu, Y-J. IPC: professional type 1 interferon-producing cells and plasmacytoid dendritic cell precursors. Annu. Rev. Immunol. 23, 275-306 (2005).

9. Bell, D., Young, J.W. \& Banchereau, J. Dendritic cells. Adv. Immunol. 72, 255-324 (1999).

10. Romani, N., Holzmann, S., Tripp, C.H., Koch, F. \& Stoitzner, P. Langerhans cells - dendritic cells of the epidermis. APMIS 111, 725-740 (2003).

11. Schuler, G. \& Steinman, R.M. Murine epidermal Langerhans cells mature into potent immunostimulatory dendritic cells in vitro. J. Exp. Med. 161, 526-546 (1985).
12. Turnbull, E.L., Yrlid, U., Jenkins, C.D. \& Macpherson, G.G. Intestinal dendritic cell subsets: differential effects of systemic TLR4 stimulation on migratory fate and activation in vivo. J. Immunol. 174, 1374-1384 (2005).

13. Hemmi, H. et al. Skin antigens in the steady state are trafficked to regional lymph nodes by transforming growth factor- $\beta 1$-dependent cells. Int. Immunol. 13, 695-704 (2001).

14. Henri, S. et al. The dendritic cell populations of mouse lymph nodes. J. Immunol. 167, 741-748 (2001).

15. Jakob, T., Ring, J. \& Udey, M.C. Multistep navigation of Langerhans/ dendritic cells in and out of the skin. J. Allergy Clin. Immunol. 108, 688696 (2001).

16. Worbs, T. et al. Oral tolerance originates in the intestinal immune system and relies on antigen carriage by dendritic cells. J. Exp. Med. 203, 519-527 (2006).

17. Wilson, N.S. et al. Most lymphoid organ dendritic cell types are phenotypically and functionally immature. Blood 102, 2187-2194 (2003).

18. Vremec, D., Pooley, J., Hochrein, H., Wu, L. \& Shortman, K. CD4 and CD8 expression by dendritic cell subtypes in mouse thymus and spleen. J. Immunol. 164, 2978-2986 (2000).

19. Vremec, D. et al. The surface phenotype of dendritic cells purified from mouse thymus and spleen: investigation of the CD8 expression by a subpopulation of dendritic cells. J. Exp. Med. 176, 47-58 (1992).

20. Crowley, M., Inaba, K., Witmer-Pack, M. \& Steinman, R.M. The cell surface of mouse dendritic cells: FACS analyses of dendritic cells from different tissues including thymus. Cell. Immunol. 118, 108-125 (1989).

21. Lahoud, M.H. et al. Signal regulatory protein molecules are differentially expressed by CD8- dendritic cells. J. Immunol. 177, 372-382 (2006).

22. Edwards, A.D. et al. Relationships among murine CD11 chigh dendritic cell subsets as revealed by baseline gene expression patterns. J. Immunol. 171, 47-60 (2003).

23. Proietto, A.I. et al. Differential production of inflammatory chemokines by murine dendritic cell subsets. Immunobiology 209, 163-172 (2004).

24. Hochrein, H. et al. Differential production of IL-12, IFN- $\alpha$, and IFN-ã by mouse dendritic cell subsets. J. Immunol. 166, 5448-5455 (2001).

25. Reis e Sousa, C. et al. In vivo microbial stimulation induces rapid CD40 ligand-independent production of interleukin 12 by dendritic cells and their redistribution to T-cell areas. J. Exp. Med. 186, 1819-1829 (1997).

26. den Haan, J.M., Lehar, S.M. \& Bevan, M.J. CD8 ${ }^{+}$but not CD8 ${ }^{-}$dendritic cells cross-prime cytotoxic T cells in vivo. J. Exp. Med. 192, 1685-1696 (2000).

27. Pooley, J.L., Heath, W.R. \& Shortman, K. Cutting edge: intravenous soluble antigen is presented to CD4T cells by CD8- dendritic cells, but cross-presented to CD8T cells by CD8 ${ }^{+}$dendritic cells. J. Immunol. 166, 5327-5330 (2001).

28. Belz, G.T. et al. Cutting edge: conventional CD $8 \alpha^{+}$dendritic cells are generally involved in priming CTL immunity to viruses. J. Immunol. 172, 1996-2000 (2004)

29. Steinman, R.M., Pack, M. \& Inaba, K. Dendritic cells in the T-cell areas of lymphoid organs. Immunol. Rev. 156, 25-37 (1997).

30. Agger, R. et al. Two populations of splenic dendritic cells detected with M342, a new monoclonal to an intracellular antigen of interdigitating dendritic cells and some B lymphocytes. J. Leukoc. Biol. 52, 34-42 (1992).

31. De Smedt, T. et al. Regulation of dendritic cell numbers and maturation by lipopolysaccharide in vivo. J. Exp. Med. 184, 1413-1424 (1996).

32. Metlay, J.P. et al. The distinct leukocyte integrins of mouse spleen dendritic cells as identified with new hamster monoclonal antibodies. J. Exp. Med. 171, 1753-1771 (1990).

33. Belz, G.T. et al. The $\mathrm{CD} 8^{+}$dendritic cell is responsible for inducing peripheral self-tolerance to tissue-associated antigens. J. Exp. Med. 196, 1099-1104 (2002).

34. Xu, Y., Zhan, Y., Lew, A.M., Naik, S.H. \& Kershaw, M.H. Differential development of murine dendritic cells by GM-CSF versus Flt3 ligand has implications for inflammation and trafficking. J. Immunol. 179, 7577-7584 (2007).

35. Hikino, H. et al. GM-CSF-independent development of dendritic cells from bone marrow cells in the GM-CSF-receptor-deficient mouse. Transplant. Proc. 32, 2458-2459 (2000).

36. Vremec, D. et al. The influence of granulocyte/macrophage colonystimulating factor on dendritic cell levels in mouse lymphoid organs. Eur. J. Immunol. 27, 40-44 (1997).

37. Wiktor-Jedrzejczak, W. et al. Total absence of colony-stimulating factor 1 in the macrophage-deficient osteopetrotic (op/op) mouse. Proc. Natl. Acad. Sci. USA 87, 4828-4832 (1990). 
38. McKenna, H.J. et al. Mice lacking Flt3 ligand have deficient hematopoiesis affecting hematopoietic progenitor cells, dendritic cells, and natural killer cells. Blood 95, 3489-3497 (2000).

39. Laouar, Y., Welte, T., Fu, X.Y. \& Flavell, R.A. STAT3 is required for Flt3Ldependent dendritic cell differentiation. Immunity 19, 903-912 (2003).

40. Brasel, K., De Smedt, T., Smith, J.L. \& Maliszewski, C.R. Generation of murine dendritic cells from flt3-ligand-supplemented bone marrow cultures. Blood 96, 3029-3039 (2000).

41. Naik, S.H. et al. Cutting edge: generation of splenic $\mathrm{CD}^{+}$and $\mathrm{CD}^{-}$ dendritic cell equivalents in Fms-like tyrosine kinase 3 ligand bone marrow cultures. J. Immunol. 174, 6592-6597 (2005).

42. Naik, S.H. et al. Development of plasmacytoid and conventional dendritic cell subtypes from single precursor cells derived in vitro and in vivo. Nat. Immunol. 8, 1217-1226 (2007).

43. Ginhoux, F. et al. Langerhans cells arise from monocytes in vivo. Nat. Immunol. 7, 265-273 (2006).

44. Merad, M. et al. Langerhans cells renew in the skin throughout life under steady-state conditions. Nat. Immunol. 3, 1135-1141 (2002).

45. Pelayo, R. et al. Derivation of 2 categories of plasmacytoid dendritic cells in murine bone marrow. Blood 105, 4407-4415 (2005).

46. Kamath, A.T., Henri, S., Battye, F., Tough, D.F. \& Shortman, K. Developmental kinetics and lifespan of dendritic cells in mouse lymphoid organs. Blood 100, 1734-1741 (2002).

47. Kabashima, K. et al. Intrinsic lymphotoxin- $\beta$ receptor requirement for homeostasis of lymphoid tissue dendritic cells. Immunity 22, 439-450 (2005).

48. Waskow, C. et al. The receptor tyrosine kinase Flt3 is required for dendritic cell development in peripheral lymphoid tissues. Nat. Immunol. 9, 676-683 (2008).

49. Liu, K. et al. Origin of dendritic cells in peripheral lymphoid organs of mice. Nat. Immunol. 8, 578-583 (2007).

50. Leon, B. et al. Dendritic cell differentiation potential of mouse monocytes: monocytes represent immediate precursors of $\mathrm{CD}^{-}{ }^{-}$and $\mathrm{CD}^{+}$splenic dendritic cells. Blood 103, 2668-2676 (2004).

51. Naik, S.H. et al. Intrasplenic steady-state dendritic cell precursors that are distinct from monocytes. Nat. Immunol. 7, 663-671 (2006).

52. Kondo, M. et al. Biology of hematopoietic stem cells and progenitors: implications for clinical application. Annu. Rev. Immunol. 21, 759-806 (2003).

53. Akashi, K., Traver, D., Miyamoto, T. \& Weissman, I.L. A clonogenic common myeloid progenitor that gives rise to all myeloid lineages. Nature 404, 193-197 (2000).

54. Kondo, M., Weissman, I.L. \& Akashi, K. Identification of clonogenic common lymphoid progenitors in mouse bone marrow. Cell 91, 661-672 (1997).

55. Inaba, K. et al. Granulocytes, macrophages, and dendritic cells arise from a common major histocompatibility complex class II-negative progenitor in mouse bone marrow. Proc. Natl. Acad. Sci. USA 90, 3038-3042 (1993).

56. MacDonald, K.P. et al. The colony-stimulating factor 1 receptor is expressed on dendritic cells during differentiation and regulates their expansion. J. Immunol. 175, 1399-1405 (2005).
57. Caux, C. et al. CD34+ hematopoietic progenitors from human cord blood differentiate along two independent dendritic cell pathways in response to GM-CSF+TNF $\alpha$. J. Exp. Med. 184, 695-706 (1996).

58. Ardavin, C., Wu, L., Li, C.L. \& Shortman, K. Thymic dendritic cells and T cells develop simultaneously in the thymus from a common precursor population. Nature 362, 761-763 (1993).

59. Karsunky, H. et al. Developmental origin of interferon- $\alpha$-producing dendritic cells from hematopoietic precursors. Exp. Hematol. 33, 173-181 (2005).

60. Manz, M.G., Traver, D., Miyamoto, T., Weissman, I.L. \& Akashi, K. Dendritic cell potentials of early lymphoid and myeloid progenitors. Blood 97, 3333-3341 (2001).

61. Wu, L. et al. Development of thymic and splenic dendritic cell populations from different hemopoietic precursors. Blood 98, 3376-3382 (2001).

62. D'Amico, A. \& Wu, L. The early progenitors of mouse dendritic cells and plasmacytoid predendritic cells are within the bone marrow hemopoietic precursors expressing Flt3. J. Exp. Med. 198, 293-303 (2003).

63. Onai, N., Obata-Onai, A., Schmid, M.A. \& Manz, M.G. Flt3 in regulation of type I interferon-producing cell and dendritic cell development. Ann. NY Acad. Sci. 1106, 253-261 (2007).

64. Onai, N. et al. Identification of clonogenic common Flt3 ${ }^{+} \mathrm{M}-\mathrm{CSFR}^{+}$ plasmacytoid and conventional dendritic cell progenitors in mouse bone marrow. Nat. Immunol. 8, 1207-1216 (2007).

65. Fogg, D.K. et al. A clonogenic bone marrow progenitor specific for macrophages and dendritic cells. Science 311, 83-87 (2006).

66. Varol, C. et al. Monocytes give rise to mucosal, but not splenic, conventional dendritic cells. J. Exp. Med. 204, 171-180 (2007).

67. Corcoran, L. et al. The lymphoid past of mouse plasmacytoid cells and thymic dendritic cells. J. Immunol. 170, 4926-4932 (2003).

68. Shigematsu, H. et al. Plasmacytoid dendritic cells activate lymphoidspecific genetic programs irrespective of their cellular origin. Immunity 21, 43-53 (2004).

69. Yang, G.X. et al. Generation of functionally distinct B-lymphocytes from common myeloid progenitors. Clin. Exp. Immunol. 150, 349-357 (2007).

70. Fancke, B., Suter, M., Hochrein, H. \& O'Keeffe, M. M-CSF: a novel plasmacytoid and conventional dendritic cell poietin. Blood 111, 150-159 (2008).

71. Maraskovsky, E. et al. Dramatic increase in the numbers of functionally mature dendritic cells in Flt3 ligand-treated mice: multiple dendritic cell subpopulations identified. J. Exp. Med. 184, 1953-1962 (1996).

72. Pulendran, B. et al. Distinct dendritic cell subsets differentially regulate the class of immune response in vivo. Proc. Natl. Acad. Sci. USA 96, 10361041 (1999).

73. Brawand, P. et al. Murine plasmacytoid pre-dendritic cells generated from Flt3 ligand-supplemented bone marrow cultures are immature APCs. J. Immunol. 169, 6711-6719 (2002).

74. Onai, N., Obata-Onai, A., Tussiwand, R., Lanzavecchia, A. \& Manz, M.G. Activation of the Flt3 signal transduction cascade rescues and enhances type I interferon-producing and dendritic cell development. J. Exp. Med. 203, 227-238 (2006). 